\title{
Strength vs. temporal-order information in delayed-matching-to-sample performance by monkeys
}

\author{
THOMAS J. REYNOLDS and DOUGLAS L. MEDIN \\ The Rockefeller University, New York, New York 10021
}

\begin{abstract}
The usual procedure in delayed-matching-to-sample (DMTS) experiments is that the most recently presented sample stimulus is correct on choice tests. The present paper reports two experments in which the first of two samples was always correct. Theories based on trace strength, such as the Roberts and Grant trace strength competition model, predict that such a procedure will yield below chance performance, because on the average the more recently presented stimulus will have the greater trace strength. In contrast, D'Amato and others have proposed that DMTS performance is mediated by a temporal discrimination process and, since either stimulus may act as $\mathrm{S}+$ in a discrimination problem, monkeys should be capable of learning to choose either the second or the first of two sample stimuli. Performance did not exceed chance in either experiment. The generality of these results is tentatively explored.
\end{abstract}

The increasing number of experiments concerned with animal memory (e.g., D'Amato, 1973; Honig \& James, 1971; Jarrard, 1971; Medin, Roberts, \& Davis, 1976) has stimulated a corresponding interest in theories of animal memory. The paradigm used in much of this research, the delayed-matching-tosample procedure (DMTS), has been the basis for two major theoretical developments, the independenttrace-strength and competition model (Roberts \& Grant, 1974, 1976) and the temporal discrimination hypothesis (D'Amato, 1973). Although derived from quite different assumptions, each model provides an excellent account of basic DMTS phenomena, including delay, sample set size, and proactive interference effects (D'Amato, 1973; Mason \& Wilson, 1974; Mishkin \& Delacour, 1975; Roberts \& Grant, 1974, 1976; Worsham, 1975). Indeed, it has been difficult to develop experiments for which the theories offer contrasting expectations. The current exploratory work focused on a task which provided a strong contrast between a strength theory and a temporal discrimination theory of DMTS.

Before discussing the theories in detail, it will be helpful to describe the DMTS paradigm more completely. In this task, a sample stimulus (A) is presented and responded to, and following a delay interval, a

This research was supported by U.S. Public Health Service Grant MH 25134 to D.L.M. and Postdoctoral Fellowship MH 07551 to T.J.R. The authors wish to thank John Parkinson and Robert Hendersen for their helpful comments on an earlier draft of this paper. Reprints may be obtained from the first author, the Center of Alcohol Studies, Rutgers University, Busch Campus, New Brunswick, New Jersey 08903. Douglas L. Medin's current address is: Department of Psychology, University of Illinois, Champaign, Illinois 61820 . choice is offered between the sample (A) and a new stimulus (C), with the sample correct and rewarded $(A+C)$. Responses to sample presentations are typically rewarded in experiments with primates tested on a Wisconsin General Test Apparatus (WGTA) (e.g., Mason \& Wilson, 1974; Mishkin \& Delacour, 1975), while rewards are usually available only on choice tests when automated procedures and an operant chamber are used (e.g., D'Amato, 1973; Worsham, 1975). If the $C$ stimulus on a test is totally novel, DMTS performance is better than when a small pool of sample stimuli is used, since the $\mathrm{C}$ stimulus may have served as the sample just a trial or so earlier (Mason \& Wilson, 1974; Medin, 1974; Mishkin \& Delacour, 1975; Worsham, 1975).

A common variation on the basic DMTS paradigm is to present sample $\mathrm{A}$, followed by a second sample (B), and after a delay interval, to give a choice between $\mathrm{A}$ and $\mathrm{B}$ with $\mathrm{B}$ correct and rewarded $(\mathrm{AB}+)$. Both strength and temporal discrimination theories predict performance on $\mathrm{A}+\mathrm{C}$ control trials to be better than on $\mathrm{AB}+$ interference trials, a result which has been obtained consistently (Grant \& Roberts, 1973; Jarvik, Goldfarb, \& Carley, 1969; Roberts \& Grant, 1976).

The independent-trace-strength and competition model was developed by Roberts and Grant (1974, 1976) in a series of experiments studying pigeon short-term memory. In their formulation, memory trace strength for a stimulus accumulates as a negatively accelerated function of presentation duration and decays as a negatively accelerated function of delay. Successive stimulus presentations are encoded as separate memory traces, which grow and decay independently. At the time of a test, behavior is 
assumed to be controlled by the competition between these traces, with the stimulus associated with greater strength being chosen.

The Roberts and Grant theory is consistent with the basic delay, sample-set size, and proactive interference effects mentioned earlier. Accuracy is predicted to decline with delay, since it is assumed that trace strength decays in a negatively accelerated fashion with time. Performance should also vary with sample set size because the smaller the sample set, the more likely it is that the incorrect alternative has recently appeared as the sample and been correct, thereby accumulating additional trace strength. When the incorrect stimulus serves as a sample within a trial sequence, as it does on $\mathrm{AB}+$ interference trials, the strength of $A$ will be high and $A$ will compete strongly with $\mathrm{B}$ on the $\mathrm{AB}$ choice test.

The temporal discrimination hypothesis (D'Amato, 1973) offers a distinctly different theoretical approach to DMTS. One way to characterize simple DMTS is to observe that while a single trial consists of the discrete sequence sample-delay-choice: sample vs. new stimulus, over the course of many trials, individual samples recede into a set of sample events organized in time. Thus, the correct solution over the long run could be stated as "choose the stimulus most recently presented" rather than "choose the sample." In other words, DMTS procedures can be viewed as requiring animals to make relative recency judgments. As the delay interval increases, this temporal-order discrimination becomes more difficult and accuracy declines. Sample-set size effects occur because with smaller sample sets, the incorrect stimulus alternative will have served as the sample more recently. $A+C$ control trials are again predicted to be easier than $\mathrm{AB}+$ interference trials, since the presentation of both test alternatives immediately prior to the delay in the interference condition substantially increases the difficulty of the relative recency judgment. Finally, both temporal discrimination and the Roberts and Grant theories predict that very little if any proactive interference will be found on two-sample trials, if the second sample is tested against a new stimulus (i.e., A-B-delay-B + C). This result has also been obtained (Grant, 1975; Zentall \& Hogan, 1974, 1977).

Although temporal discrimination and the Roberts and Grant trace strength theories offer the same expectations in a variety of experimental situations, the difference in their underlying mechanisms suggests a means of contrasting them. Consider a situation where sample $A$ is presented, followed by sample B, and after a delay, an $A B$ choice test is given, but stimulus $A$ is correct and rewarded $(A+B)$. According to strength theory, if $A$ and $B$ are presented for equal durations, the trace strength for stimulus $A$ will be less than or equal to that of stimulus $B$ at the time of an A+B test, since $A$ will have had a longer time to decay than $\mathbf{B}$.
Given that the strength of $B$ must be at least as great as that of $A$, performance on $A+B$ tests is predicted not to exceed chance. This prediction follows directly from the Roberts and Grant theory, and holds for strength theories in general which assume that the stimulus associated with the greater strength will be chosen.'

In contrast, if DMTS can be rightly viewed as a discrimination task, it should be arbitrary whether the first or the second sample is designated $\mathrm{S}+$ or $\mathrm{S}-$. Thus, the temporal discrimination hypothesis predicts that accuracy on an $A+B$ test will exceed chance as behavior comes under the control of experimenter-defined contingencies. To be sure, animals with experience having the second sample as $\mathrm{S}+$ might well start out below chance, but performance should improve with further training. Thus, above-chance performance on $\mathbf{A}+\mathbf{B}$ tests is perfectly compatible with a temporal discrimination theory but inconsistent with trace strength theory.

Although the strength theory prediction that A + B performance will not exceed chance seems straightforward, two potentially mitigating factors need to be addressed. First, one would want to insure that successful A + B performance did not derive from the animals employing the rule of always avoiding the $B$ stimulus on a test. In addition, one would want some assurance that animals paid equal (or nearly equal) attention to each of the two sample stimuli, because attending to stimulus $A$ and ignoring $B$ would create a situation where $A$ would have greater trace strength than B. Therefore, in the present study, additional conditions were added to rule out strategies based on avoiding the most recent (strongest) stimulus and to provide an independent index of the trace strengths associated with the $\mathrm{A}$ and $\mathrm{B}$ stimuli.

\section{EXPERIMENT 1}

The various trial types used in the first study are schematically presented in Table 1 . It is important to note that new stimuli were used on every trial so that the particular A stimulus on an A + B trial, for example, did not appear on any other trial sequence. The rationale for using trial-unique stimuli is to reduce between-trial interactions, thereby eliminating the necessity for balancing the frequency and recency of rewards associated with each stimulus.

Table 1

Trial Sequence for Each Condition

\begin{tabular}{|c|c|c|c|c|c|}
\hline Trial Type & & Sample(s) & & Delay* & Test \\
\hline $\begin{array}{l}A+B \\
A \text { Control }\end{array}$ & $\begin{array}{l}\text { At+ } \\
\text { At }\end{array}$ & $12 \mathrm{sec}$ & $\mathrm{B}+$ & 12,24 & $\mathrm{~A}+\mathrm{B}$ \\
\hline & $\mathrm{A}+$ & $12 \mathrm{sec}$ & $B+$ & $\begin{array}{l}27,39 \\
12,24\end{array}$ & $\begin{array}{l}A+C \\
A+C\end{array}$ \\
\hline B Control & & & $\mathrm{B}+$ & 12,24 & $\mathrm{~B}+\mathrm{C}$ \\
\hline PI & $\mathrm{A}^{+}$ & $12 \mathrm{sec}$ & B+ & 12,24 & $B+C$ \\
\hline
\end{tabular}

*In seconds. 
Such a procedure might also serve to increase the salience of temporal-order information. From common experience, it seems that the temporal sequence of very frequently occurring events is more difficult to remember precisely than the ordering of relatively novel events. Since each stimulus appeared on the average only once in 5 days, temporal-order information might well be more readily accessible in the current paradigm.

The trial sequence involving the critical $A+B$ test is at the top of Table 1 , and the other trial sequences provide important control conditions. The A control, $B$ control, and PI (proactive interference) trials serve to rule out the simple strategy of avoiding the most recently presented sample stimulus. Further, the PI trials provide an index of the extent to which stimulus $B$ is attended to when it is the second of two samples. Neither trace strength nor temporal discrimination theory predicts a difference between B control and PI trials. The RI (retroactive interference) condition, when compared with the A controls, provides an index of how much the presentation of $B$ interferes with the strength of A. Again, neither theory expects any difference. The RI and PI trials taken together provide a measure of the trace strengths of $A$ and $B$ that can be used to evaluate the critical $A+B$ tests. For example, if $A+B$ performance exceeded chance while the PI and RI conditions were equally good, the possibility that $A+B$ performance was based on some process either inordinately strengthening $A$ or weakening B could be discounted.

\begin{abstract}
Method
Subjects. The subjects were four pigtailed monkeys (Macaca nemestrina), two rhesus monkeys (Macaca mulatta), and one cynomolgus monkey (Macaca fascicularis). All were 5- to 8-yearold, jungle-born females which had been in learning and memory experiments for the last 2-3 years. This experience included testing on various types of discrimination learning problems, including DMTS tasks in which a single sample was presented, rewarded, and was correct on the choice test. The rhesus and pigtailed monkeys had also run in experiments in which the sample might not be rewarded, in which case it was incorrect on the choice test. These monkeys had also been tested with two and three stimuli presented within a trial, with the correct choice determined by the reward status of the last sample. They were maintained on a 12-h/12-h light-dark cycle initiated at $0700 \mathrm{~h}$, and were fed lab chow daily after testing.
\end{abstract}

Apparatus. The monkeys were tested in a darkened room that had white-noise masking. The Wisconsin General Test Apparatus (WGTA) was lighted by two 15 -W fluorescent bulbs, and the three foodwells on the gray formboard were spaced $15 \mathrm{~cm}$, center to center. The stimulus pool consisted of 364 junk objects, each of which was spray-painted one of 14 colors.

Procedure. The pigtails, cynomolgus, and one of the rhesus monkeys were run 5 days/week, 30 trials/day, for 40 days. A session consisted of 12 single-sample and 18 two-sample trials (see Table 1) intermixed according to one of six random orders. On control or single-sample trials (either A control or B control), the sample stimulus was presented over the center foodwell, and the monkey displaced it for a raisin reward. The opaque WGTA screen was then lowered and, after a delay of $12,24,27$, or $39 \mathrm{sec}$, the sample and a new object (C) were presented over the side foodwells. Choice of the stimulus which had served as the sample was rewarded by a raisin $(A+C$ or $B+C)$. Since pilot work had indicated that the monkeys averaged $3 \mathrm{sec}$ to respond to a sample stimulus, A control trial delays were 27 or $39 \sec$ (i.e., $12+3+12$ $=27$ or $12+3+24=39$ ).

On two-sample trials (A+B, PI, and RI sequences), one sample was presented, the opaque screen was lowered, and $12 \mathrm{sec}$ later another sample was given over the center foodwell. A choice test was given after either a 12 - or a $24-\mathrm{sec}$ delay during which the screen was also closed. In the PI condition, the second sample was tested against a new stimulus $(B+C)$, while in the RI sequence, the first sample appeared on the choice test $(A+C)$. In the critical $A+B$ condition, both samples were presented, with choice of the first correct and rewarded $(A+B)$. Each Condition by Delay combination appeared three times daily.

The second rhesus monkey received a slight modification of the above procedure. Control delays were either 12 or $27 \sec (=12+3$ +12 ), and interference delays were always $12 \mathrm{sec}$. Unlike the main experimental group, which received the same number of trials/ condition/day, this animal was given $12 \mathrm{~A}+\mathrm{B}$ trials, $4 \mathrm{PI}, 4 \mathrm{RI}$, and 4 control trials for a total of 24 trials/day for 30 days. This manipulation resulted in half of the daily sessions consisting of A + B trials and half PI, RI, and control trials. For all animals, trials were run in one of six quasi-random orders constructed so that the correct object was presented equally of ten in the right and left positions, and never occupied the same position for more than four consecutive trials. A noncorrection procedure was employed, and the ITI was a constant $18 \mathrm{sec}$. New objects were used on each trial, and the entire stimulus pool was reshuffled after each five sessions.

\section{Results}

Table 2 presents mean proportion correct for each condition subdivided into 10-day practice blocks (180 observations/point). Performance on the A + B test was initially below chance and did not exceed chance after $\mathbf{4 0}$ days of practice. In contrast, performance in the other four conditions was initially above chance and improved substantially with practice. The RI and PI conditions proved to be no more difficult than their corresponding controls.

The data were analyzed by a Trial Types by Practice Blocks within-subjects ANOVA. There were significant main effects of both factors $[F(9,45)=31.17$ for Trial Types and $F(3,15)=8.90$ for Practice Blocks, ps $<.01$ ], with performance on the two

Table 2

Mean Proportion Correct for Each Condition in Experiment 1

\begin{tabular}{lccccccc}
\hline & & Sample-Test & \multicolumn{4}{c}{ 10-Day Practice Blocks } \\
Trial Type & Delay* & Interval* & 1 & 2 & 3 & 4 & $\overline{\mathrm{X}}$ \\
\hline \multirow{2}{*}{ A+B } & 12 & 27 & .39 & .38 & .37 & $.42 \dagger$ & .39 \\
& 24 & 39 & $.44 \dagger$ & $.40 \dagger$ & $.48 \dagger$ & $.43 \dagger$ & .44 \\
A Control & 27 & 27 & .70 & .85 & .80 & .86 & .80 \\
& 39 & 39 & .69 & .75 & .81 & .85 & .77 \\
RI & 12 & 27 & .68 & .79 & .85 & .89 & .80 \\
& 24 & 39 & .76 & .82 & .82 & .80 & .80 \\
B Control & 12 & 12 & .74 & .85 & .88 & .93 & .85 \\
& 24 & 24 & .71 & .87 & .88 & .88 & .84 \\
PI & 12 & 12 & .75 & .86 & .91 & .92 & .86 \\
& 24 & 24 & .71 & .83 & .88 & .88 & .83 \\
\hline
\end{tabular}

*In seconds. $\dagger$ Not significantly different from chance, based on a binomial model, $p<.01$. 
A + B conditions reliably worse than all other trial types, which did not differ from each other, and significant improvement occurring between the first and second 10-day periods (Newman-Keuls, ps $<.05$ ). The Trial Types by Practice interaction approached significance $[F(27,135)=1.43, .10>p>.05]$, since A + B performance did not change with practice, while all other conditions showed reliable improvement across test blocks (Newman-Keuls, ps $<.05$ ).

Delay effects were tested with separate Trial Type by Delay ANOVAs for the A control, RI, and A + B condition (i.e., 27- and 39-sec sample-test intervals) and the B control and PI condition (i.e., 12- and 24-sec sample-test intervals). There was a main effect of Trial Type in the first analysis $[\mathrm{F}(2,10)=33.73$, $p<.01$, but no Delay effect or interaction. The $A+B$ condition was poorer than either A control or RI, which did not differ (Newman-Keuls, $p<.01$ ). There was a significant Delay effect in the second ANOVA $[\mathrm{F}(1,5)=10.50, \mathrm{p}<.05]$, with accuracy decreasing between 12 and $24 \mathrm{sec}$, but no Trial Type effect or interaction. Thus, neither the PI nor the RI conditions differed from their appropriate controls at either sample-test interval, while the $\mathrm{A}+\mathrm{B}$ test was more difficult than its $A$ control at both delays. Three animals were significantly below chance on A + B trials, while the remaining three responded at chance. In sharp contrast, most animals were significantly better than chance on all other conditions by the end of 20 days, and all were above chance on the other trial types by the end of the experiment (ps $<.05$ ), binomial test). Thus, the poor performance on $\mathrm{A}+\mathrm{B}$ trials cannot be attributed to any overall difficulty with DMTS for these subjects. In fact, the Pearson product-moment correlation between $\mathrm{A}+\mathrm{B}$ performance and the other conditions combined was $-.87(\mathrm{df}=6, \mathrm{p}<.01)$, indicating that the best monkeys overall tended to be worst on $A+B$ trials.

The second rhesus, run with half $A+B$ trials per day, responded in a similar manner. After 30 days of testing, her performance was above chance for the control and interference conditions (proportion correct: $12-\mathrm{sec}$ control $=.78, \mathrm{PI}=.77 ; 24-\mathrm{sec}$ control $=.77, \mathrm{RI}=.60$ ) and below chance for the $\mathrm{A}+\mathrm{B}$ trials (proportion correct $=.36 ; \mathrm{ps}<.05$ ).

\section{Discussion}

The results of the first experiment were consistent with trace strength theory and failed to give support to the idea that DMTS performance in these animals was based on a temporal discrimination. In particular, the asymptotic chance-level responding and lack of improvement with practice on the $\mathrm{A}+\mathrm{B}$ condition conformed to the rather counterintuitive prediction of trace strength theory that this trial sequence would not be mastered.
These results, of course, are not definitive, inasmuch as their generality is an open question. To begin with, it could be argued that since our monkeys had had considerable DMTS experience in which B was correct on $\mathrm{AB}$ choice tests, 40 days of practice was insufficient to alter their response strategy. Although there is no definite way to resolve this issue, it is worth pointing out that, in general, monkeys adjust quickly to procedural changes. For example, we have switched these same monkeys from a procedure where the sample was always rewarded and correct to one in which it was not rewarded on half the trials and the sample was correct on the choice test only if it had been rewarded. We observed clear negative transfer at the beginning of testing, but very rapid improvement and complete adaptation within 15-20 days. The quick improvement in performance on the other trial types and the absence of any practice effect on the $A+B$ tests, however, indicates that a simple preexperimental bias was not operating.

A related argument arises from the trial-type structure used in the first experiment. On the one hand, every trial sequence other than the PI condition could be solved by always choosing the first-presented sample. Alternatively, on every sequence other than $\mathrm{A}+\mathrm{B}$, the correct response was to choose the most recently presented stimulus. Since the PI condition proved to be no harder than controls while the $A+B$ test was clearly most difficult, it is apparent that the monkeys tended to choose recent stimuli rather than first-presented stimuli. However, since the novel objects on $\mathrm{A}+\mathrm{C}$ and $\mathrm{B}+\mathrm{C}$ tests were only relatively new (i.e., appearing on the average once every 5 days), the task may have been highly confusing in that it required the monkeys to choose the more recent stimulus on four of the five trial types and the less recent stimulus on the A + B test. In other words, stimuli of intermediate recency were always correct, remote stimuli were always incorrect, and the most recent stimuli were sometimes correct and sometimes incorrect.

If the monkeys were, in fact, attending to temporalorder information but chose an inappropriate response rule because of the structure of Experiment 1, presenting A + B trials exclusively should produce a sudden improvement in performance, since a simple response rule (choose the earlier stimulus) would be appropriate on every trial. A second experiment was run to assess this possibility. It is important to keep in mind that positive results would be somewhat ambiguous since no controls were given in conjunction with the $\mathrm{A}+\mathrm{B}$ trials. However, if performance continued to be at chance levels, our conclusion concerning the difficulty of the $A+B$ condition would be considerably more general. 


\section{EXPERIMENT 2}

In this experiment, additional practice was given on $\mathrm{A}+\mathrm{B}$ trials as the only trial sequence. While such a design is not as well controlled as Experiment 1, our intention was to see if appropriate $A+B$ choices would develop under these simple conditions. Thus, if the description of DMTS performance as involving a temporal-order discrimination is correct, this experiment should maximize the probability that the monkeys would learn the discrimination. In contrast, if trace strength theory is the more accurate, the results of this experiment should replicate the first experiment.

\section{Method \\ Subjects and Apparatus. The three subjects in Experiment 2 were one of the four pigtailed monkeys and the rhesus and cynomolgus monkeys from the main group of Experiment 1. The details of maintenance, apparatus, and the stimulus pool were identical to Experiment 1. \\ Procedure. The monkeys were run 5 days/week, 30 trials/day for 12 days. The trial sequence was identical to the $A+B$ condi- tion in Experiment 1, with a random half being 12-sec and the other half 24-sec delays. Briefly, a sample (A) was presented and rewarded, the opaque screen was lowered to initiate a 12-sec ISI; then a second sample (B) was presented and rewarded; after a 12- or a 24-sec delay spent with the screen down, $A$ was rewarded in a choice test between $A$ and $B(A+B)$. As before, the posi- tion of the correct object was randomized but balanced within a day and an 18-sec ITI was used. New objects were given on each trial and the stimulus pool was reshuffled after five sessions.}

\section{Results}

The mean proportion correct for each delay and 4-day practice block is presented in Table $3(180 \mathrm{ob}-$ servations/point). In no case was performance significantly above chance. Analysis by a within-subjects ANOVA with 4-day Practice Blocks and Delay as factors revealed no significant main effects or interactions (ps $>.05$ ). At the level of means, chance performance was not exceeded during any practice block at either delay. In fact, mean proportion correct was significantly below chance during the second practice block for the 12-sec delay and during the first block for the 24-sec delay (ps $<.05$, binomial test). The overall performance of individual monkeys was somewhat mixed. The pigtail performed significantly below chance at both delays (.37 and .41 correct for 12- and 24-sec delays, respectively), the rhesus was

Table 3

Mean Proportion Correct for Each Condition in Experiment 2

\begin{tabular}{cccccc} 
& & \multicolumn{5}{c}{ 4-Day Practice Blocks } \\
Trial Type & Delay* & 1 & 2 & 3 & $\overline{\mathrm{X}}$ \\
\hline \multirow{2}{*}{ A+B } & 12 & $.46 \dagger$ & .40 & $.52 \dagger$ & $.46 \dagger$ \\
& 24 & .40 & $.51 \dagger$ & $.47 \dagger$ & $.46 \dagger$ \\
\hline
\end{tabular}

*In seconds. TNot significantly different from chance, based on a binomial model, $p<.01$. not different from chance at 12 -sec (.57) and below chance at 24-sec delays (.36), and the cynomolgus was just above chance (.61) at 24-sec and no different from chance at 12 -sec delays $(.44$, ps $<.05$, binomial test). Thus, the additional $360 \mathrm{~A}+\mathrm{B}$ trials in the current experiment did not yield any clear evidence of above-chance responding.

\section{GENERAL DISCUSSION}

These experiments explored a simple DMTS paradigm for which the trace strength and temporal discrimination theories made contrasting predictions. It was initially expected that the $A+B$ test would be easy for our monkeys to master, and that these results would highlight a basic shortcoming of strength theories. However, 40 days of practice yielded essentially no evidence that the $\mathrm{A}+\mathrm{B}$ problem was solvable, a result which was generally predicted by trace strength theory and not by the temporal discrimination hypothesis. This lack of improvement on A+B was accompanied by large improvements in performance in the other experimental conditions. Even the radical step of presenting only $A+B$ trials in the second experiment did not alter this picture. ${ }^{2} \mathrm{Al}$ though we still believe that monkeys can attend to and solve problems on the basis of temporal-order information, it appears that temporal order is not salient in DMTS experiments run on a WGTA.

Although temporal order does not seem to have been an influential factor in our experiments, it can be argued that temporal recency information exerted considerable control over performance. In fact, the data from the first experiment can be taken as providing some evidence against strength theories. Specifically, if mean performance on the $A$ control and $B$ control trials are used as an index of the trace strengths of the A and B stimuli, mean A+B test performance is predicted from $\mathrm{P}(\mathrm{c})_{\text {Acont }} /\left[\mathrm{P}(\mathrm{c})_{\text {Acont }}+\right.$ $\left.\mathrm{P}(\mathrm{C})_{\mathrm{Bcont}}\right]$ to be .48 correct at both delays, while .39 and .44 were observed. Thus, the monkeys tended to pick the B stimulus more often than predicted on the basis of trace strengths.

In a related experiment employing pigeons as subjects, Roberts and Grant (1974) varied the strength of the A stimulus in an $\mathrm{AB}+$ test. While the amount of interference did increase as the prior event was strengthened, performance never fell below chance, even though independent estimates of trace strengths suggested that it should have. For example, a stimulus presented for $4 \mathrm{sec}$ and tested after a 2-sec delay was chosen as accurately as a stimulus presented for $2 \mathrm{sec}$ and tested immediately. However, presenting an incorrect stimulus as the sample for $4 \mathrm{sec}$ followed by the correct stimulus for $2 \mathrm{sec}$ resulted in .70 rather than .50 accuracy on an immediate test. Apparently, some factor other than strength influenced perfor- 
mance, and Roberts and Grant suggested that pigeons have an innate tendency to approach more recent stimuli.

The recency effects observed in the Roberts and Grant study and hinted at in Experiment 1 need not, however, be based on a mechanism tied directly to temporal-order information. For example, recency preference could be predicted by a strength theory which made a distinction between short- and longterm memory, with recent events tending to displace prior events from the short-term store (e.g., Estes, 1955; Wagner, Rudy, \& Whitlow, 1973). The additional strength associated with the greater likelihood of recent events being available in short-term memory could readily mediate recency preferences.

A pair of experiments by themselves can hardly be decisive in evaluating the relative merits of such broad-scope theories as the Roberts and Grant trace competition and the D'Amato temporal discrimination theories. Certainly, the generality of the present results has not been delimited. Species, test sophistication, sample-set size, and reward schedule are just a few of the variables that might alter the present pattern of results. Of these, the reward procedure seems to us to be the most promising. Since in our experiments each sample presentation was rewarded, the monkeys may have been attending to and learning more about stimulus-reward relationships than temporal order. On the other hand, in an automated apparatus such as that used by D'Amato (1973), samples must be responded to but rewards are only given on choice tests. This procedure might well increase the salience of temporal-order information, since there are no distracting predelay rewards.

There is little question that animals can respond to temporal cues under some circumstances. For example, Devine and Jones (1975) taught two rhesus monkeys to reproduce the order of presentation of twoand three-sample sequences. In this experiment, multiple samples were given $30 \mathrm{msec}$ apart on a sample key, followed $\mathbf{3 0}$ msec later by the simultaneous display of all samples. To be correct, the monkey had to respond to the simultaneously presented stimuli in the order in which they were presented as samples. In the two-sample case, $85 \%$ accuracy was finally obtained after more than 3,000 trials, and with three samples, the monkeys were correct $65 \%$ of the time after more than 1,500 trials. While this above-chance performance suggested that the monkeys were capable of recalling temporally ordered events, the extensive practice required indicated that sequential relationships were not especially salient.

Shimp (1976) has shown that pigeons can also demonstrate recall for the temporal sequence of recent events. In his paradigm, the left and right side keys of a standard operant chamber were randomly illuminated three times in succession by an "X."
Following a delay interval, one of three colors was presented on the center key, indicating whether a peck at the position of the first, second, or third stimulus light would be rewarded. When the three samples were presented for the same duration $(.5 \mathrm{sec})$, response accuracy incresed monotonically with serial position (i.e., recency preference). However, when the first sample was presented for $10 \mathrm{sec}$ and the others for $.5 \mathrm{sec}$, the first and third items were remembered better than the second (i.e., primacy and recency effects). Thus, given the appropriate conditions, short-term memory for the first, as well as the last stimulus was demonstrated.

The present experiments do not rule out the possibility that monkeys could solve the $\mathrm{A}+\mathrm{B}$ problem if given special training procedures first. However, if we take as our goal accounting for the basic empirical relationships which have been generated by animal memory research of the last decade, then it is desirable to compare the strength vs. temporal discrimination approaches using typical procedures rather than evaluating their relative merits in special or contrived paradigms. The present experiments strongly suggest that at least with the procedures employed in our laboratory, temporal relationships do not constitute an especially potent dimension.

\section{REFERENCES}

D'Amato, M. R. Delayed matching and short-term memory in monkeys. In G. H. Bower (Ed.), The psychology of learning and motivation (Vol. 7). New York: Academic Press, 1973.

Devine, J. V., \& Jones, L. C. Matching to successive samples: A multiple-unit memory task with rhesus monkeys. Behavior Research Methods \& Instrumentation, 1975, 7, 438-440.

Estes, W. K. Statistical theory of spontaneous recovery and regression. Psychological Review, 1955, 62, 145-154.

Grant, D. S. Proactive inhibition in pigeon short-term memory. Journal of Experimental Psychology: Animal Behavior Proces ses, 1975, 104, 207-220.

Grant, D. S., \& Roberts, W. A. Trace interaction in pigeon short-term memory. Journal of Experimental Psychology, 1973, 101, 21-29.

Honig, W. K., \& James, P. H. R. (Eds.). Animal memory. New York: Academic Press, 1971.

JARRA RD, L. E. (Ed.). Cognitive processes of nonhuman primates. New York: Academic Press, 1971.

JARvik, M. E., Goldfard, T. L., \& CaRley, J. L. Influence of interference on delayed matching in monkeys. Journal of Experimental Psychology, 1969, 81, 1-6.

Mason, M., \& Wilson, M. Temporal differentiation and recognition memory for visual stimuli in rhesus monkeys. Journal of Experimental Psychology, 1974, 103, 383-390.

Medin, D. L. The comparative study of memory. Journal of Human Evolution, 1974, 3, 455-463.

Medin, D. L., Roberts, W. A., \& Davis, R. T. (Eds.). Processes of animal memory. Hillsdale, N.J: Erlbaum, 1976.

Mishkin, M., \& Delacour, J. An analysis of short-term memory in the monkey. Journal of Experimental Psychology: Animal Behavior Processes, 1975, 1, 326-334.

Roberts, W. A., \& Grant, D. S. Short-term memory in the 
pigeon with presentation time precisely controlled. Learning \& Motivation, 1974, 5, 393-408.

Roberts, W. A., \& Grant, D. S. Studies of short-term memory in the pigeon using the delayed matching to sample procedure. In D. L. Medin, W. A. Roberts, \& R. T. Davis (Eds.), Processes of animal memory. Hillsdale, N.J: Erlbaum, 1976.

Shimp, C. P. Short-term memory in the pigeon: Relative recency. Journal of the Experimental Analysis of Behavior, 1976, 25, 55-61.

Testa, T. J. Causal relationships and the acquisition of avoidance responses. Psychological Review, 1974, 81, 491-505.

Wagner, A. R., RuDy, J. W., \& Whitlow, J. W. Rehearsal in animal conditioning. Journal of Experimental Psychology, 1973, 97, 407-426.

Worsham, R. W. Temporal discrimination factors in the delayed matching-to-sample task in monkeys. Animal Learning \& Behavior, 1975, 3, 93-97.

Zentall, T. R., \& Hogan, D. E. Memory in the pigeon: Proactive inhibition in a delayed matching task. Bulletin of the Psychonomic Society, 1974, 4, 109-112.

Zentall, T. R., \& Hogan, D. E. Short-term proactive inhibition in the pigeon. Learning and Motivation, 1977, 8, 367-386.

\section{NOTE}

1. Although no one has proposed such a model, one could have a strength theory with a more flexible response rule whereby animals may respond to the stimulus associated with the lesser strength. One argument against such a theory is that organisms generally have difficulty in tasks requiring them to avoid $S+s$ or to approach $S-s$ (see Testa, 1974, for a review), which suggests that this may be a very unnatural strategy. In any event, the set of strength theories considered in this paper is restricted to those in which it is assumed that animals respond to the stimulus having the greater trace strength.

2. In a subsequent experiment, the same monkeys were given an additional $144 \mathrm{~A}+\mathrm{B}$ test trial, mixed randomly with A controls, and RI tests which varied the similarity of the sample and interfering stimulus. They were .44 correct overall on the A+B trials, while averaging .74 and .80 on the $A$ controls and RI conditions, respectively.

(Received for publication August 24, 1978; revision accepted November 22, 1978.) 